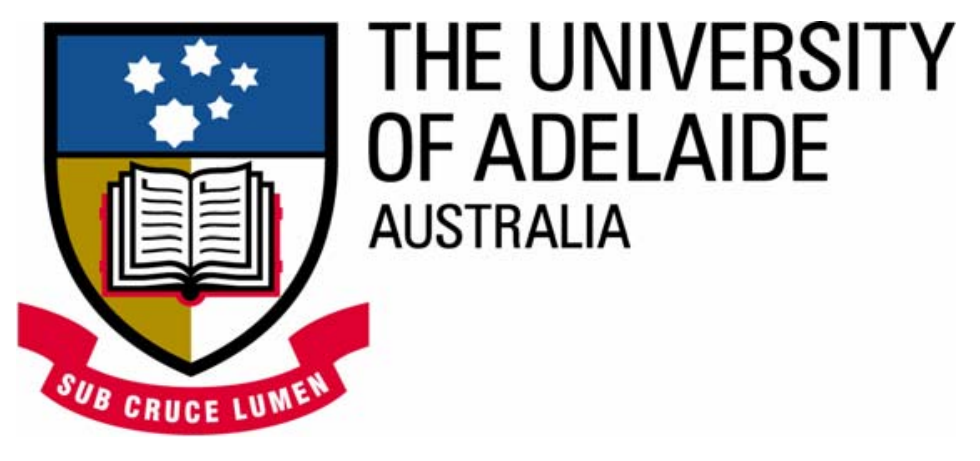

\title{
Eye safe solid state lasers for remote sensing and coherent laser radar
}

Jesper Munch, Matthew Heintze, Murray Hamilton, Sean Manning,

Y. Mao, Damien Mudge and Peter Veitch

Department of Physics

The University of Adelaide

Adelaide SA 5005

Australia 


\section{How to make a laser sensor "Eye-safe"}

Keep energy/power low

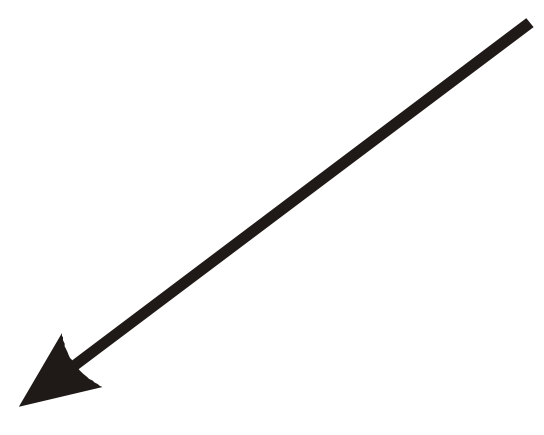

Low power laser, Large transmitted beam

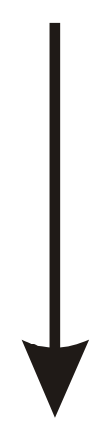

Low Duty

Cycle

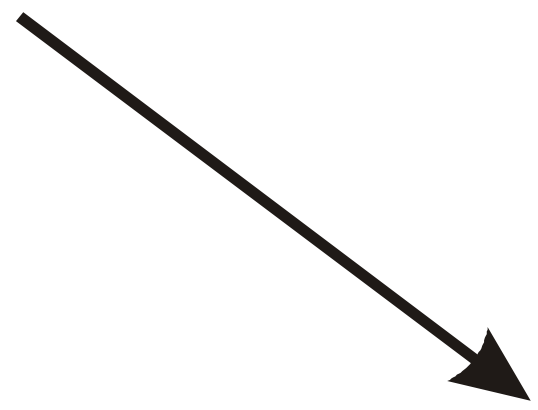

Select wavelength for maximum allowed pulse energy 


\section{Content of talk}

- Coherent eye-safe laser radar: Review of current work in Er:Yb:glass slab lasers

- Planned work in Er:Yb:YAG

- New composite slab laser design

- Eye-safe sensing at low power 


\section{Our chosen Eyesafe laser species is Erbium}

- Erbium lases at $1.5-1.6 \mu \mathrm{m}$, where laser safety allows:

- $10 \times$ the energy per pulse allowed at $2 \mu \mathrm{m}$

- 100x the energy per pulse allowed at $10 \mu \mathrm{m}$

- Allows better spatial resolution (for otherwise similar conditions)

- Can make use of available telecommunications photonic components: eg Master fiber oscillator

- BUT: it is a 3-level laser, normally in a phosphate glass host 


\section{Er:Yb energy level diagram}

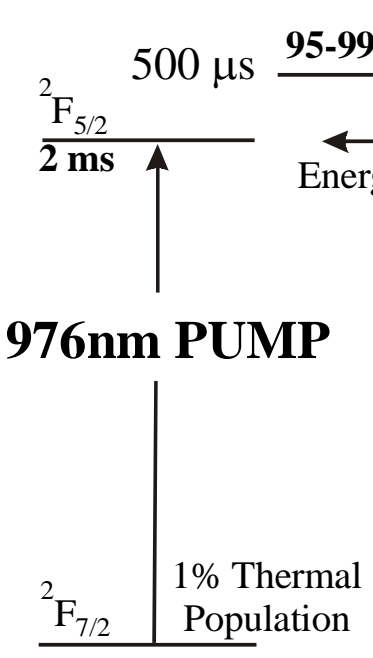

Ytterbium

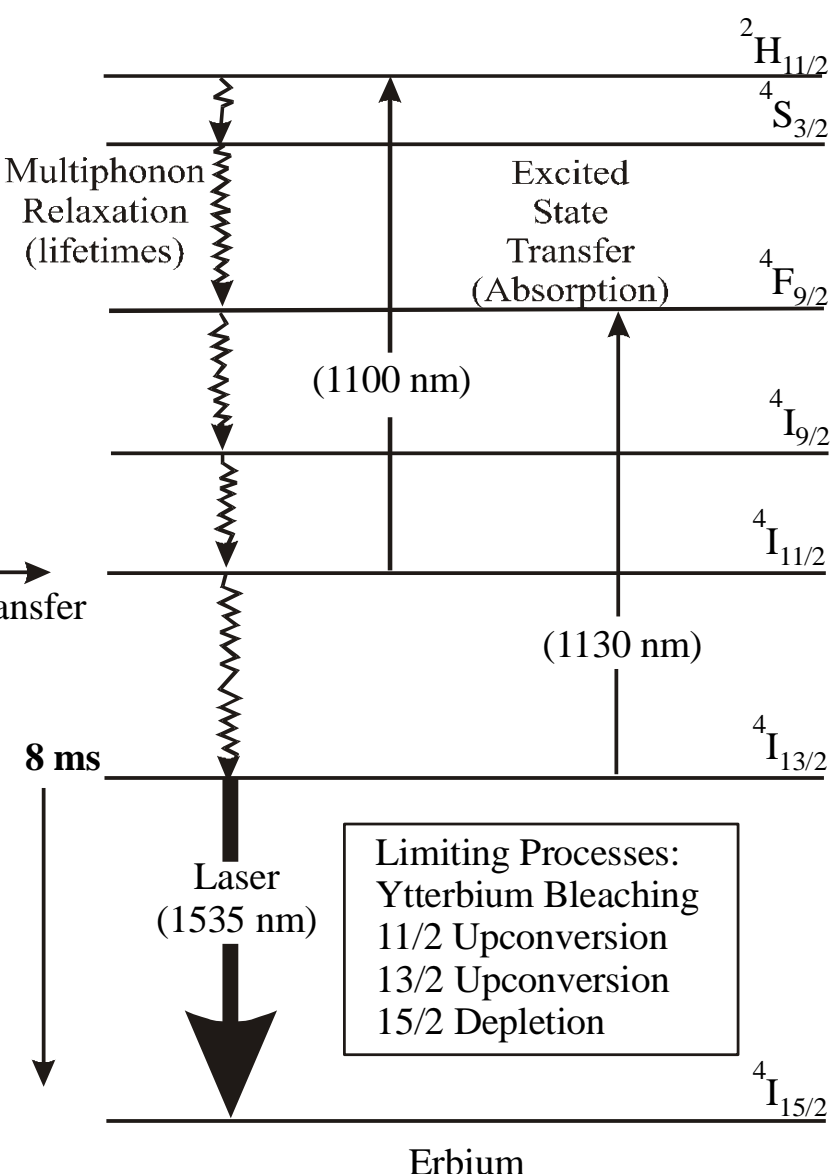




\section{Summary of Early work in Adelaide*}

- Demonstrated first injection seeding of single frequency Er:glass laser at $1.5 \mu \mathrm{m}$

- Demonstrated successful transform limited coherent Doppler measurement at $1.5 \mu \mathrm{m}$

- Initial wind sensing measurements 


\section{Coherent Laser Radar}

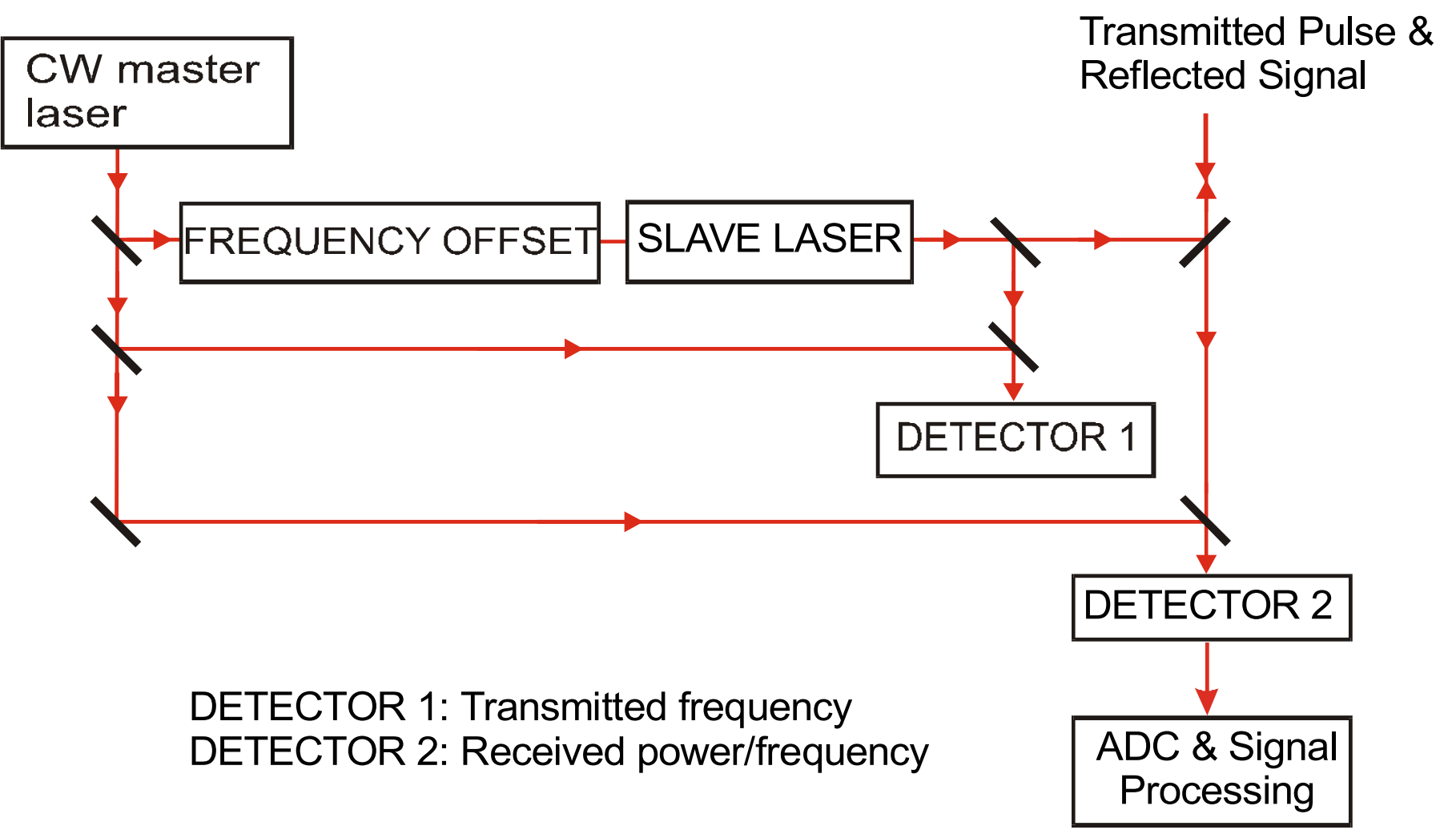




\section{First injection seeded Er:glass at $1.5 \mu \mathrm{m}$}

\section{Expanded output pulse}

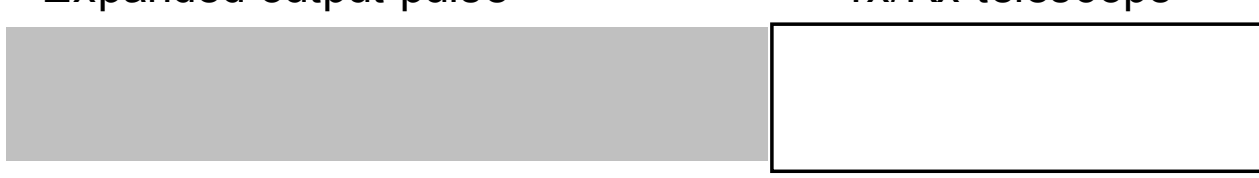

Tx/Rx telescope

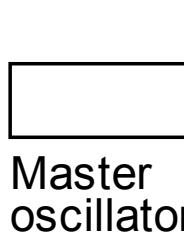

AOM Faraday isolators

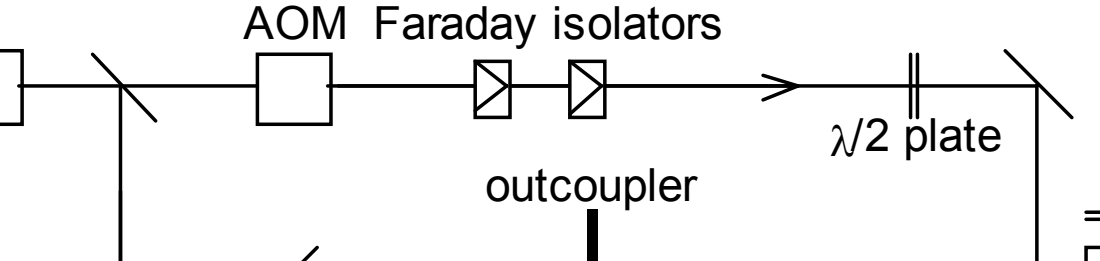

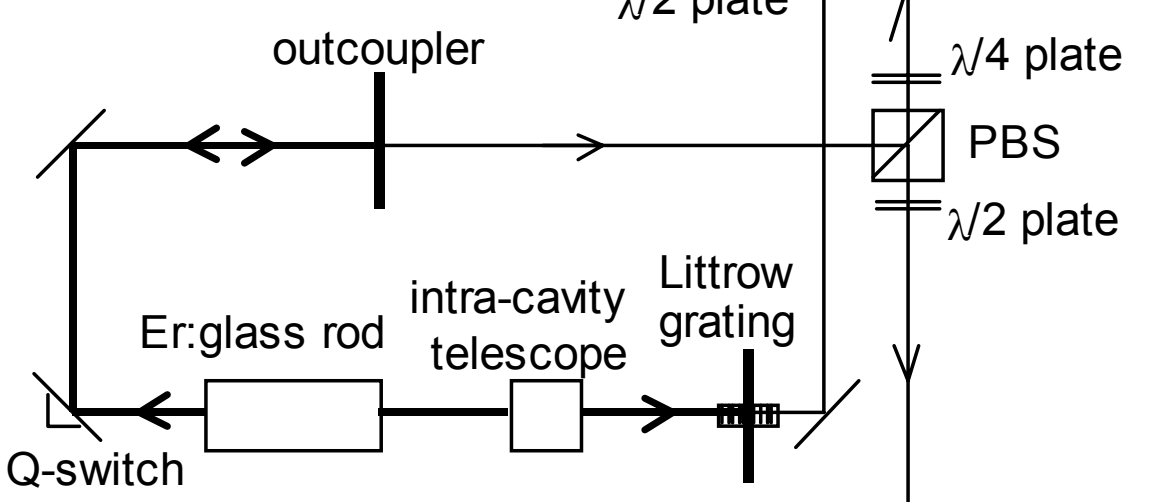

Local oscillator beam

heterodyne detector 


\section{The injection seeded, Q-switched laser produced a transform limited linewidth}
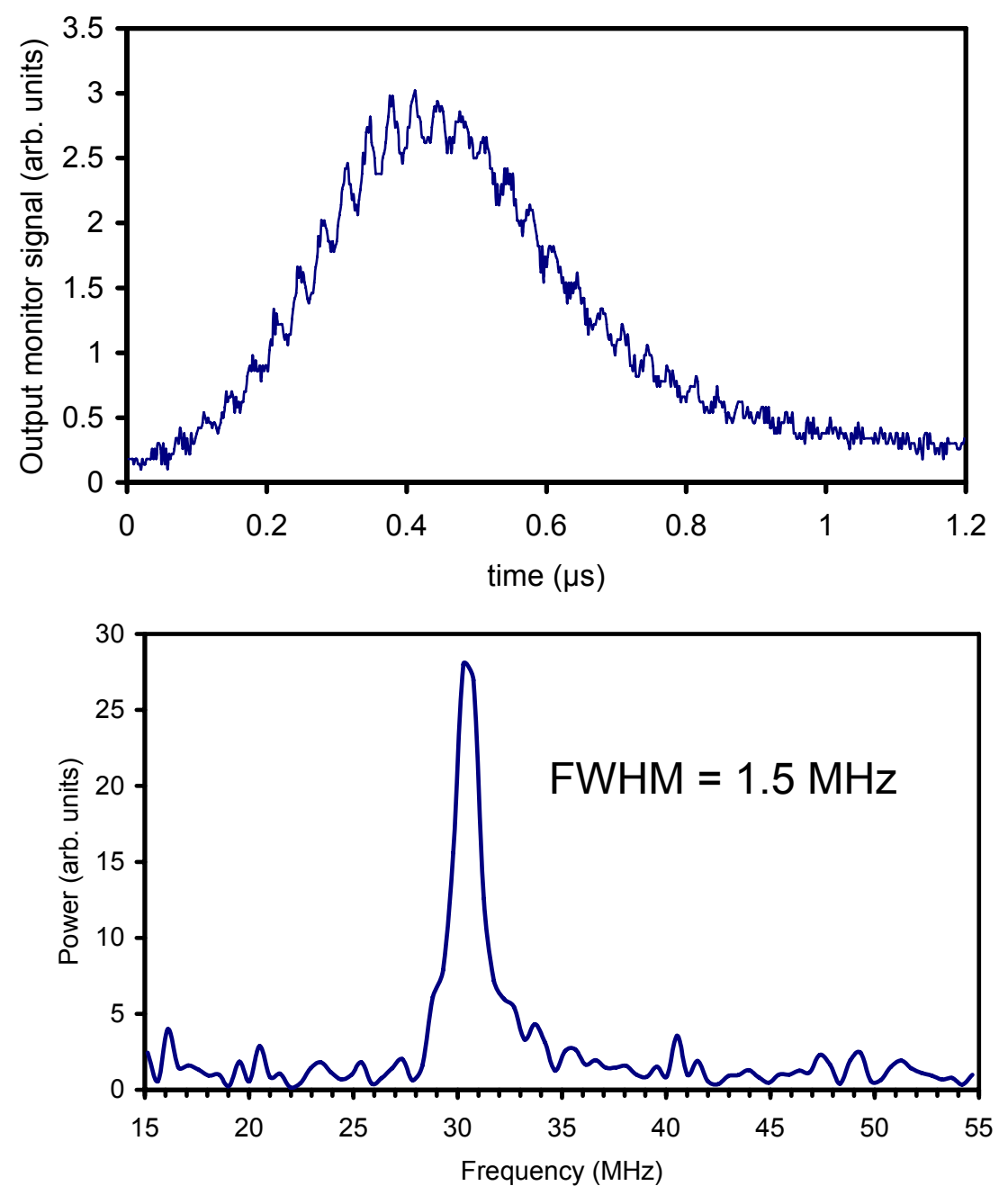


\section{We used the Er:Glass laser to make a Doppler velocity measurement of moving hard-targets}
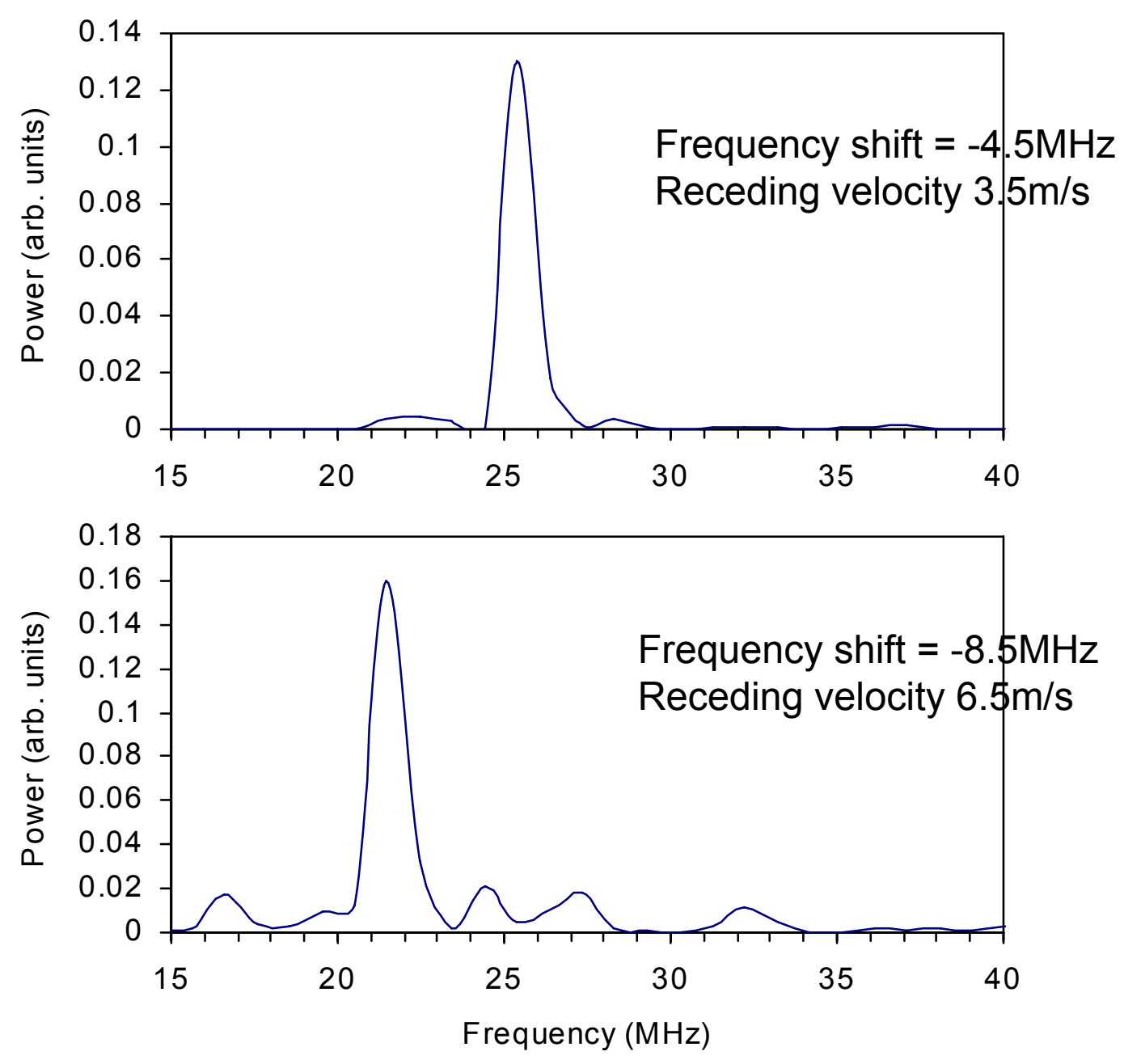


\section{Second Generation Er:Yb:Glass Slab}

- Robust laser design

- Folded, total internal reflection, zig-zag slab

- Diode laser side-pumping (Q-CW)

- Injection seeded, Q-switched ring

- Long output pulse, using new resonator design with efficient out-coupling via throttled Q-switch 


\section{Standing-wave Er:Yb:Glass slab laser}

\section{Setup}

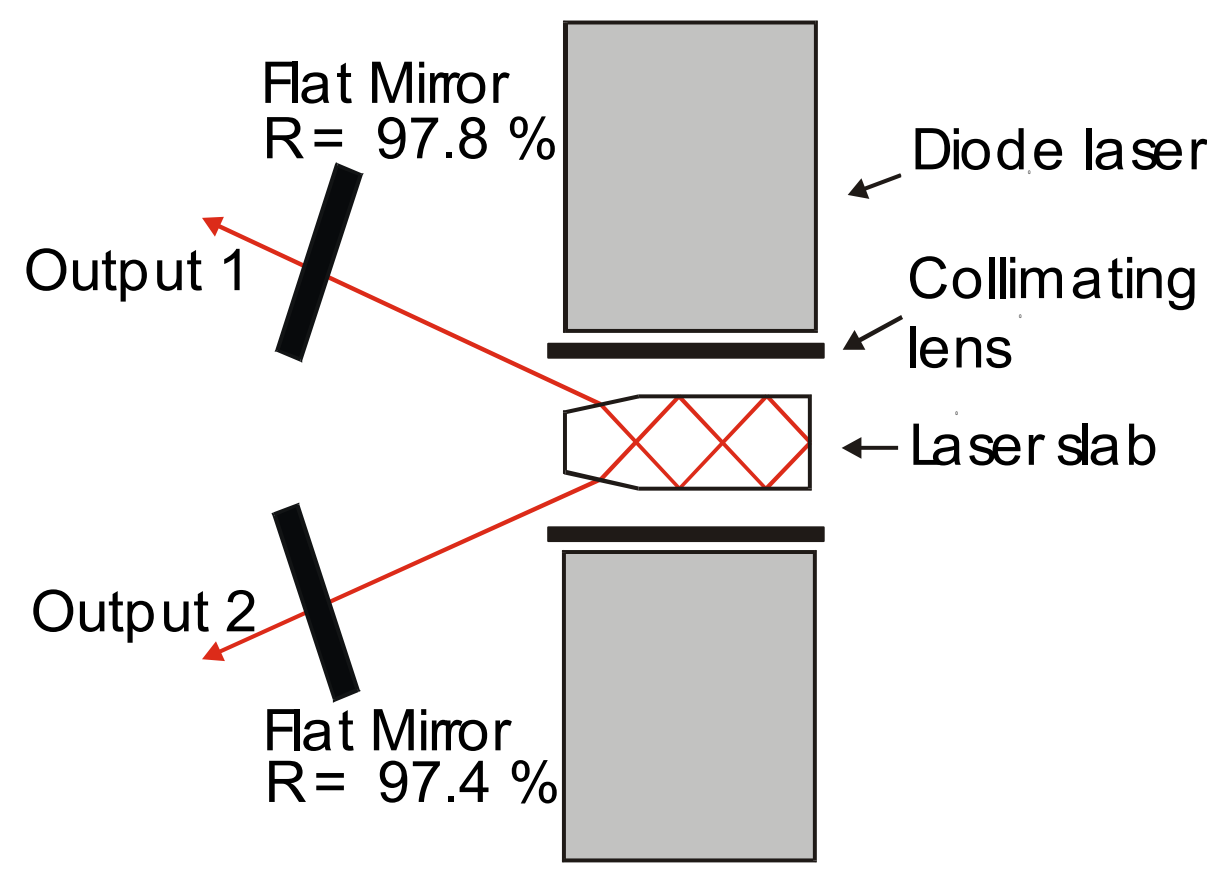

\section{Output power}

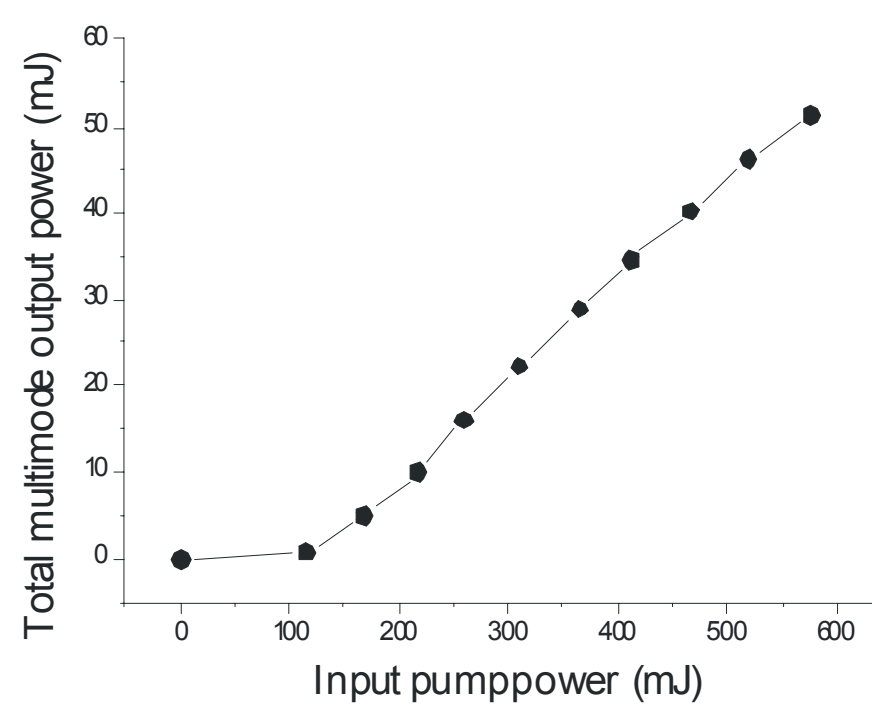




\section{Side-pumped laser head}

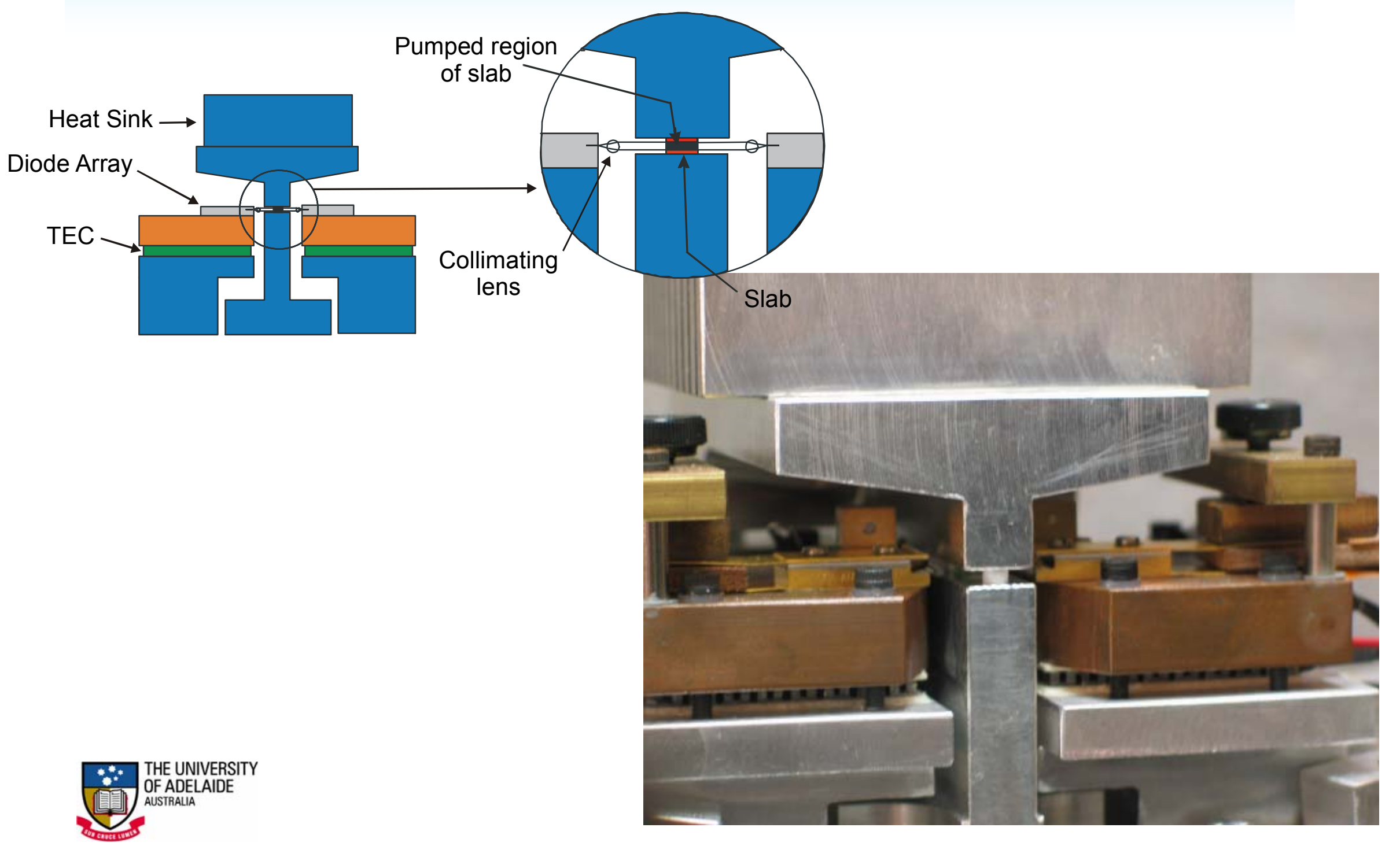




\section{Injection seeded ring resonator}

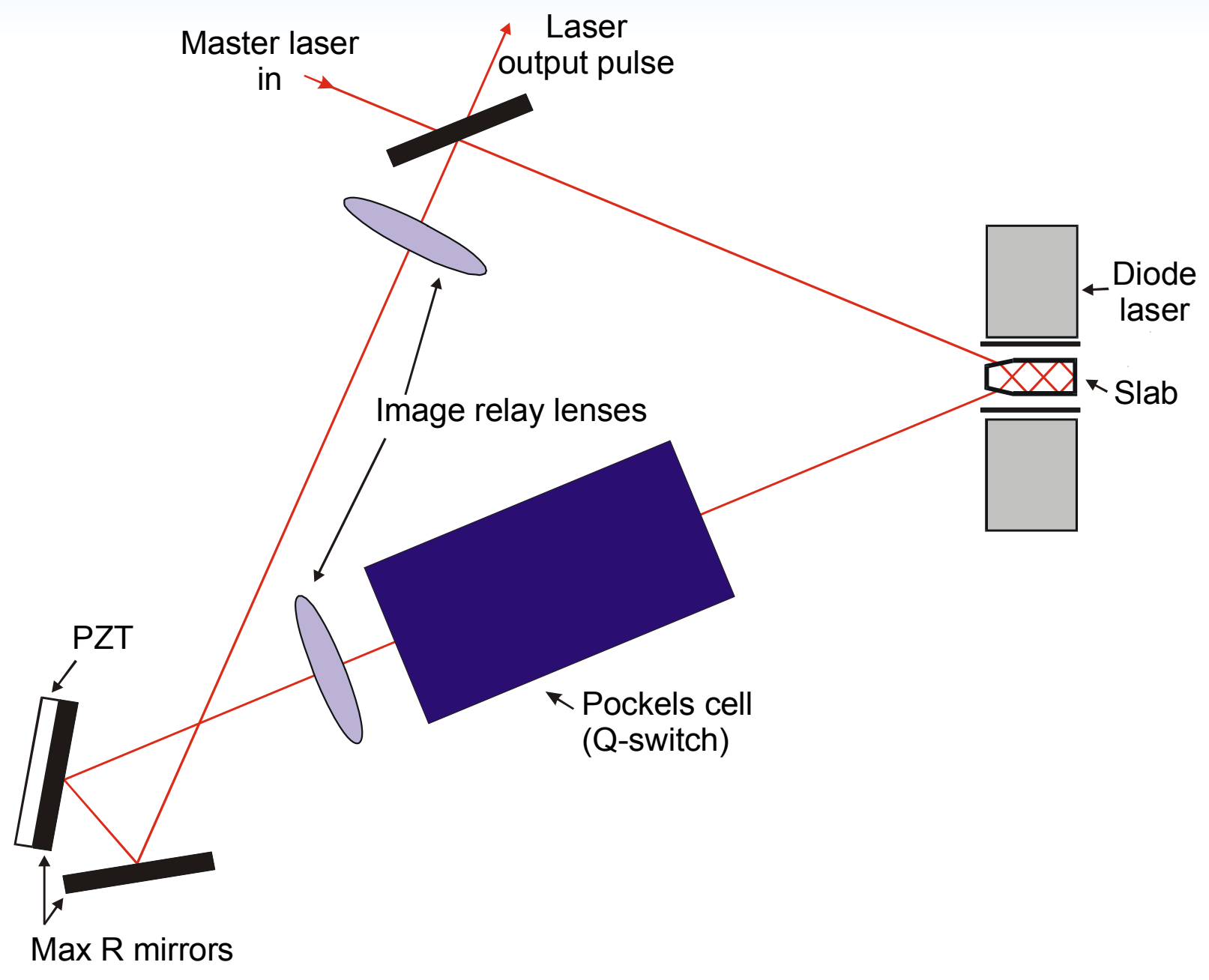




\section{Ring Oscillator Q-switch results}

Gain switched lasing ( $E=8 \mathrm{~mJ} /$ pulse)
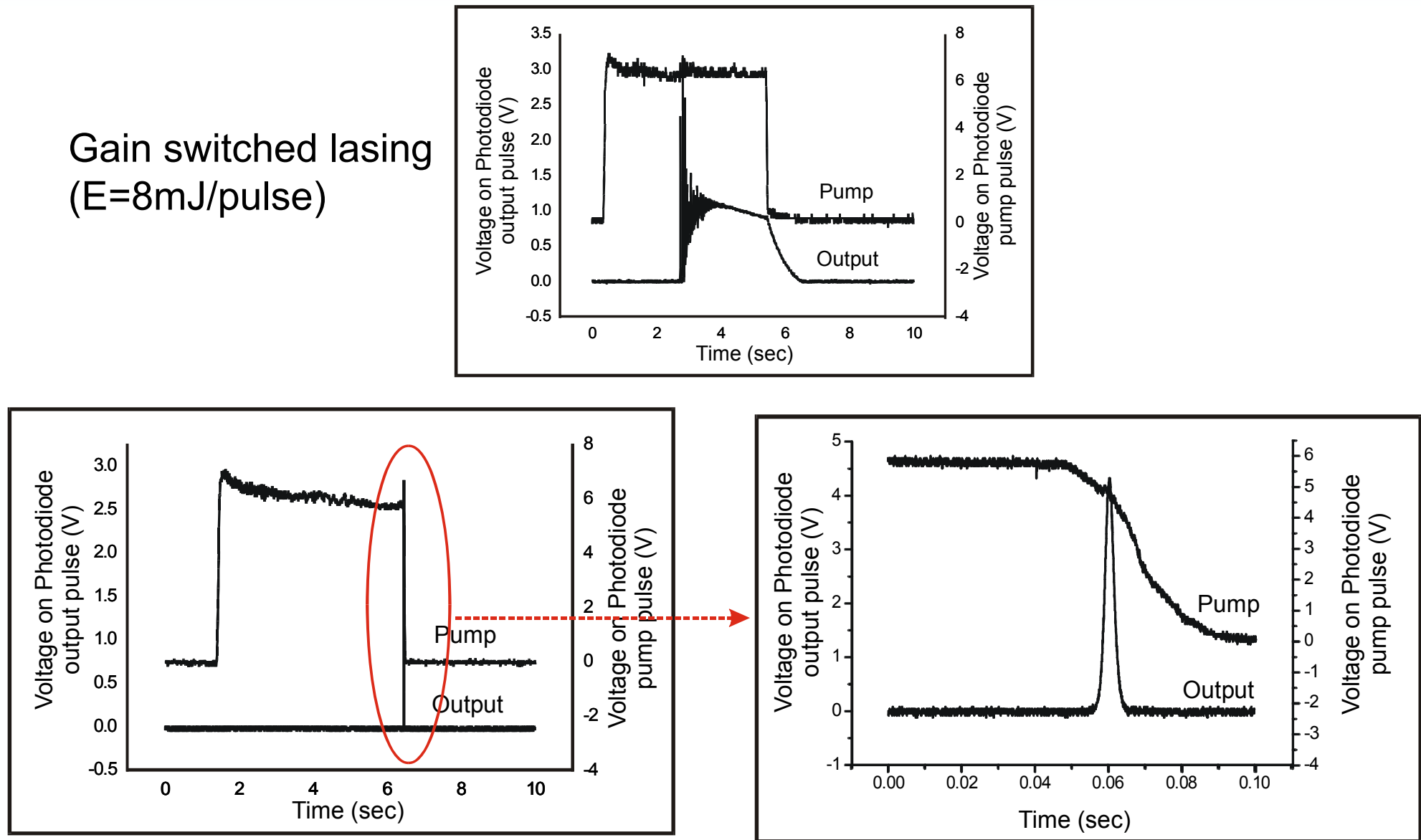

Q-switched pulse ( $E=3 \mathrm{~mJ} /$ pulse $)$ scale: ms 


\section{Current results with Er:Glass}

- Good long pulse energy in standing-wave oscillator, near TEM $\mathrm{oo}_{\mathrm{o}}$ (50mJ)

- Q-switched ring oscillator demonstrated

- Injection seeding demonstrated

\section{However:}




\section{Problems with current Er:Glass slab laser}

- Energy output limited by Er bleaching (measured)

- High intra-cavity losses in ring oscillator (Pockels cell)

- Serious thermal lensing limitations

- Optical damage of glass host

- Currently max energy per pulse Q-switched is $10 \mathrm{~mJ} /$ pulse,

but need $20-50 \mathrm{~mJ} /$ pulse raw laser output for scalable systems (eg: larger aperture, system losses)

- Pulse repetition rate will be limited by thermal effects

- Pumping limited by frequency chirp in diode-lasers used 


\section{Continuing effort in Erbium}

Two parallel approaches:

1. Improve and optimize Er:Yb:glass subject to its inherent thermal limitations.

- $\quad$ Experiments using different $\mathrm{Er}, \mathrm{Yb}$ concentrations for optimum pumping

- $\quad$ Reduce resonator losses

- Complete injection seeding characterization as laser radar

2. Investigate third generation Er:Yb:YAG 


\section{Third generation: Er:Yb:YAG at $1.645 \mu \mathrm{m}$}

- Greatly improved thermal properties of YAG host

- Better control of thermal lens

- Better efficiency (lower level has $2 \%$ population)

- Scalable to higher power, rep. rate

- Manufacture as ceramic YAG material

- Permits use of our new end-pumped composite slab geometry

- Experience from our successful Nd:YAG designs directly relevant

- But requires a new, single frequency master oscillator

- Recently demonstrated in bulk Er:Yb:YAG*

* Georgiou \& Kiriakidi, Opt. Eng., 44 Jun. 2005

$80 \mathrm{~mJ}$ output, pumped by $4.7 \mathrm{~J}$ 


\section{Scaling to higher power slabs}

High pump intensities and necessary cooling of the gain medium leads to strong thermal gradients which cause undesirable effects.

Issues

- strong thermal lensing

- change from top/bottom cooling to side cooling

- thermally induced birefringence

- use specialized pump distribution 


\section{Effect of pump profile on depolarization loss}

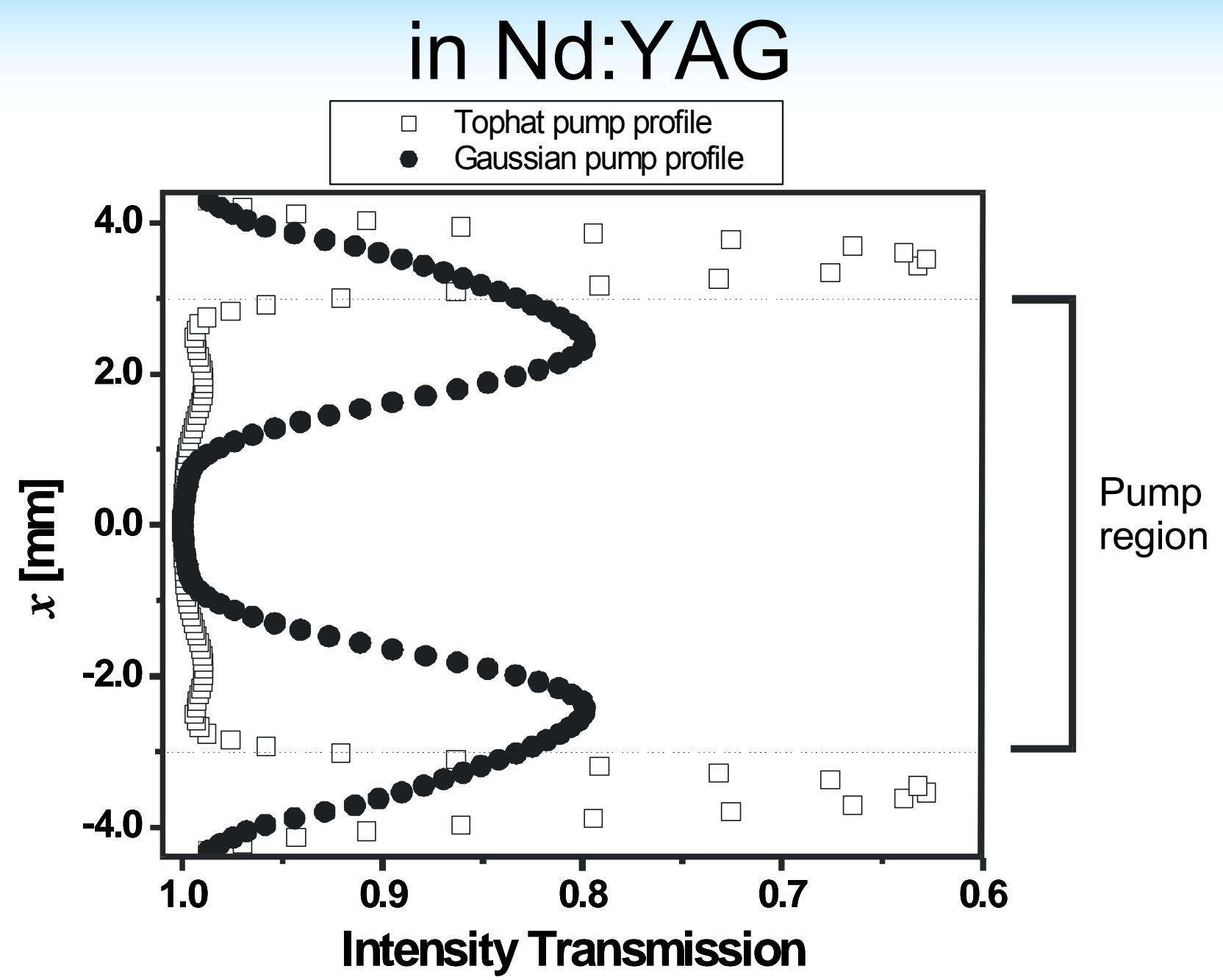

(Birefringence modeling: M. Ostermeyer) 


\section{Effect of pump profile on depolarization loss}

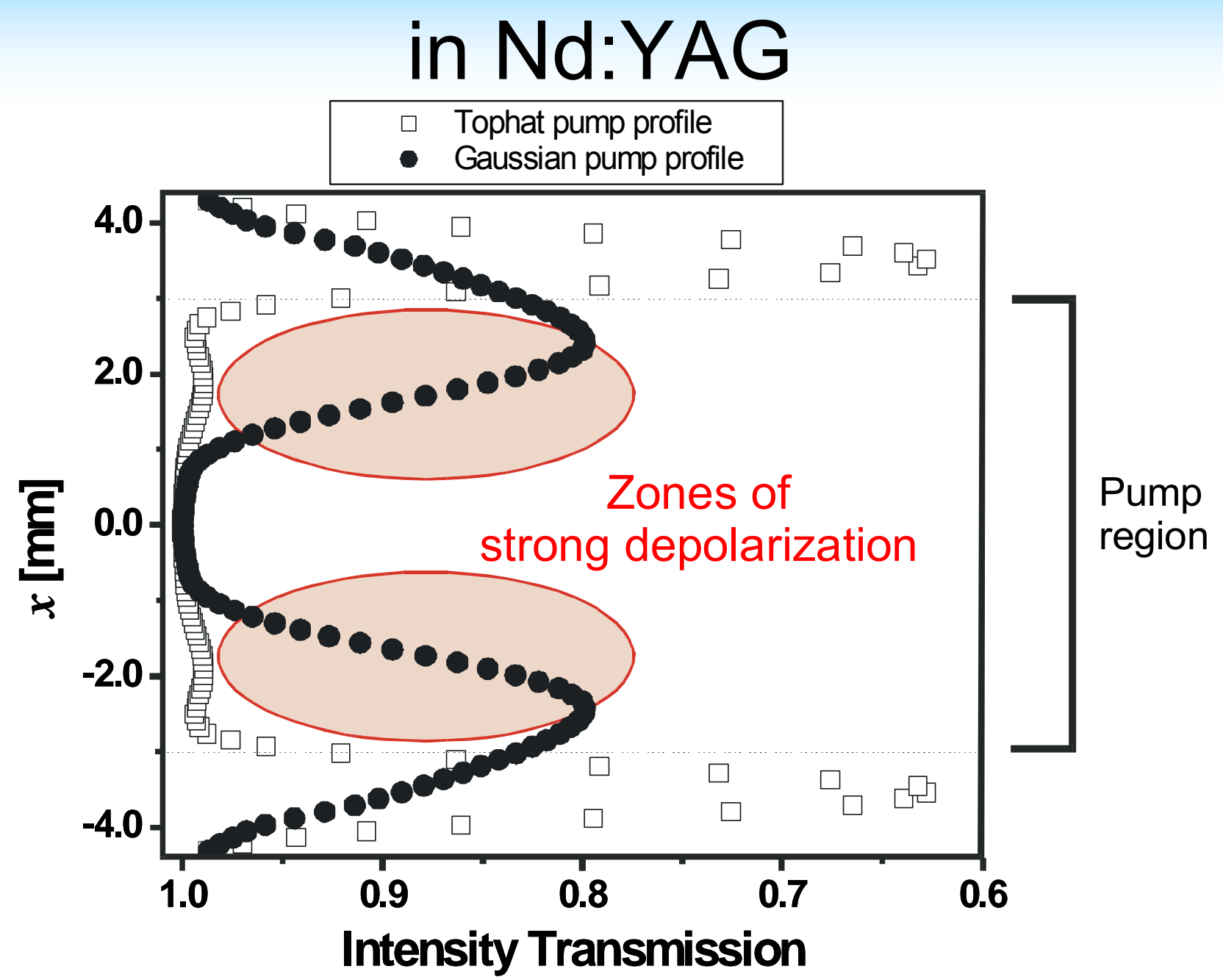

(Birefringence modeling: M. Ostermeyer) 


\section{Composite end-pumped, side-cooled folded zigzag Nd:YAG slab}
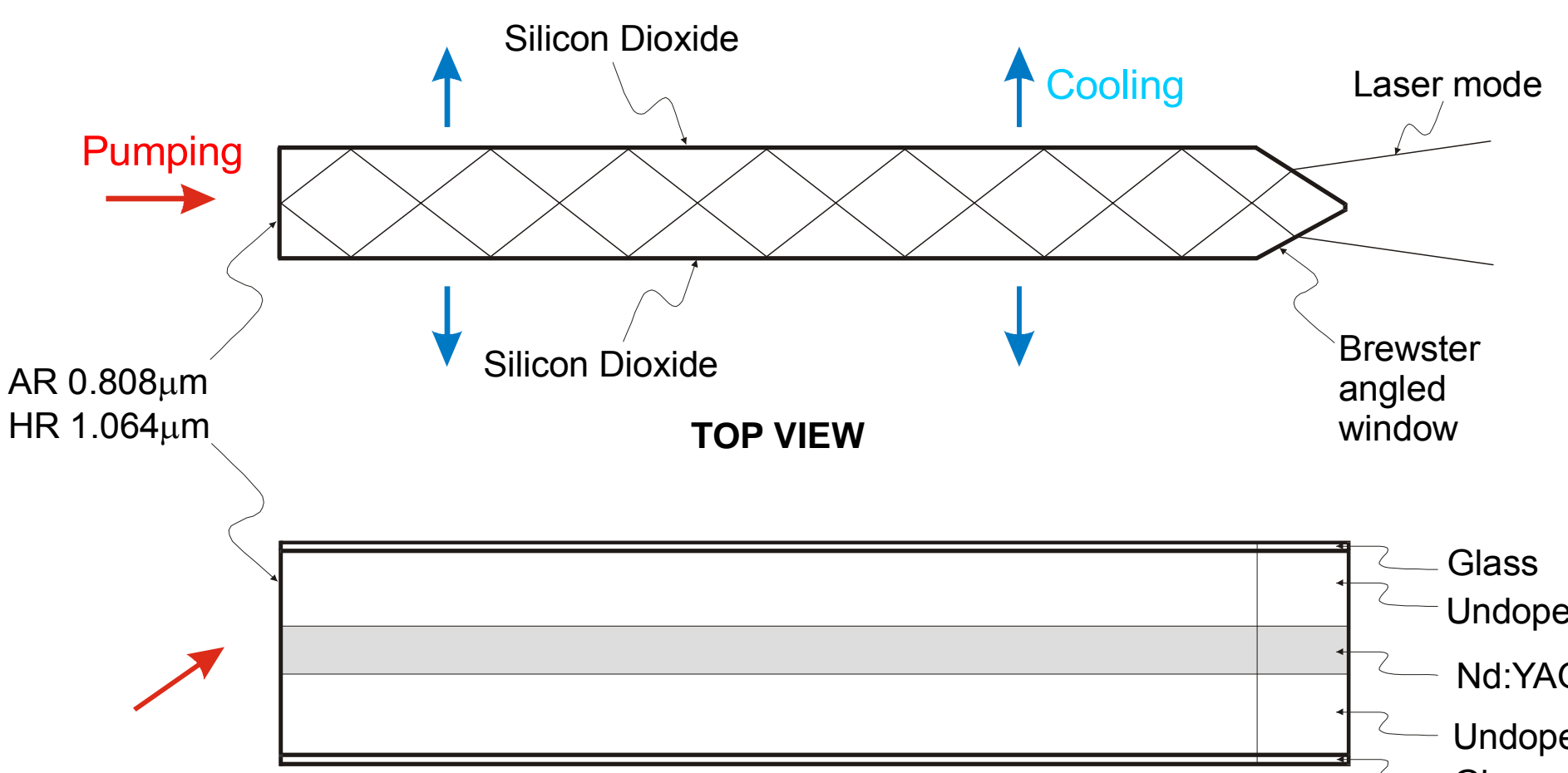

Glass

Undoped:YAG

$\mathrm{Nd}$ :YAG (0.6 at.\%)

Undoped:YAG

Glass

SIDE VIEW 


\section{Off-axis zigzag pumping}

Optical fibres

(2D array)

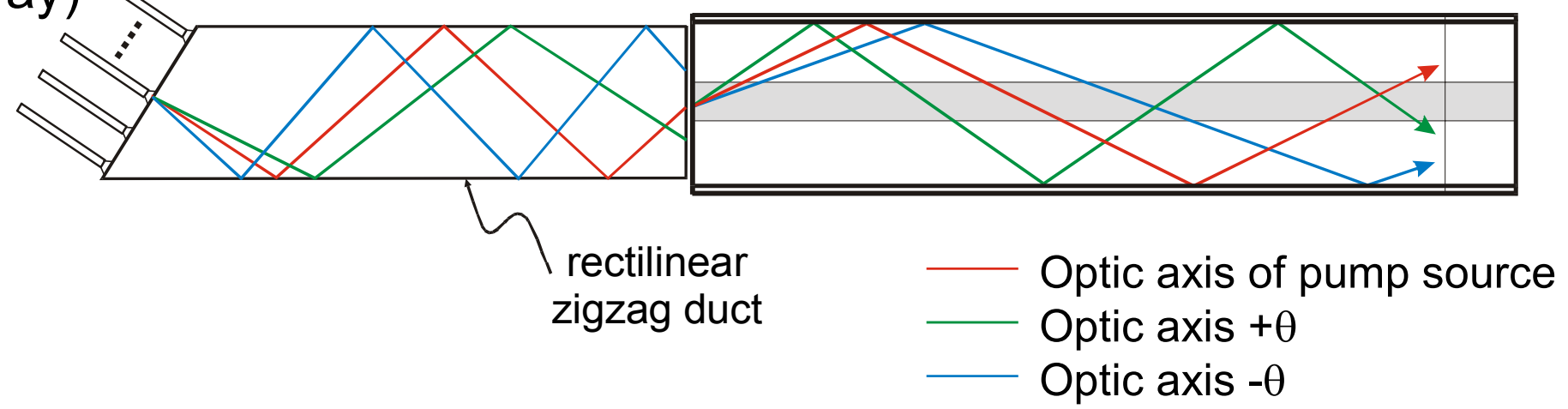

- Rectilinear zigzag duct allows pumping at normal incidence and mixes pump light prior to slab entry

- Can pump using fibers by collimated bar-stack-array, and use nonimaging lens duct

- Scalable by increasing pump power, height of doped and undoped region (mode volume) 


\section{Composite slab advantages}

- Tophat pump distribution - minimum birefringence

- Good absorption efficiency due to quasi end-pumping

- More uniform power loading within slab due to double-clad structure transporting pump light along slab before absorption

- No hard-edged apertures in vertical direction

- Large pump input aperture and acceptance angle accommodates real divergent pump sources

- Insensitive to pump beam-quality due to mixing of pump light in slab

- Undoped YAG layers produce reduced thermally induced stress

- Conduction-cooled 


\section{End view of conduction-cooled laser head}

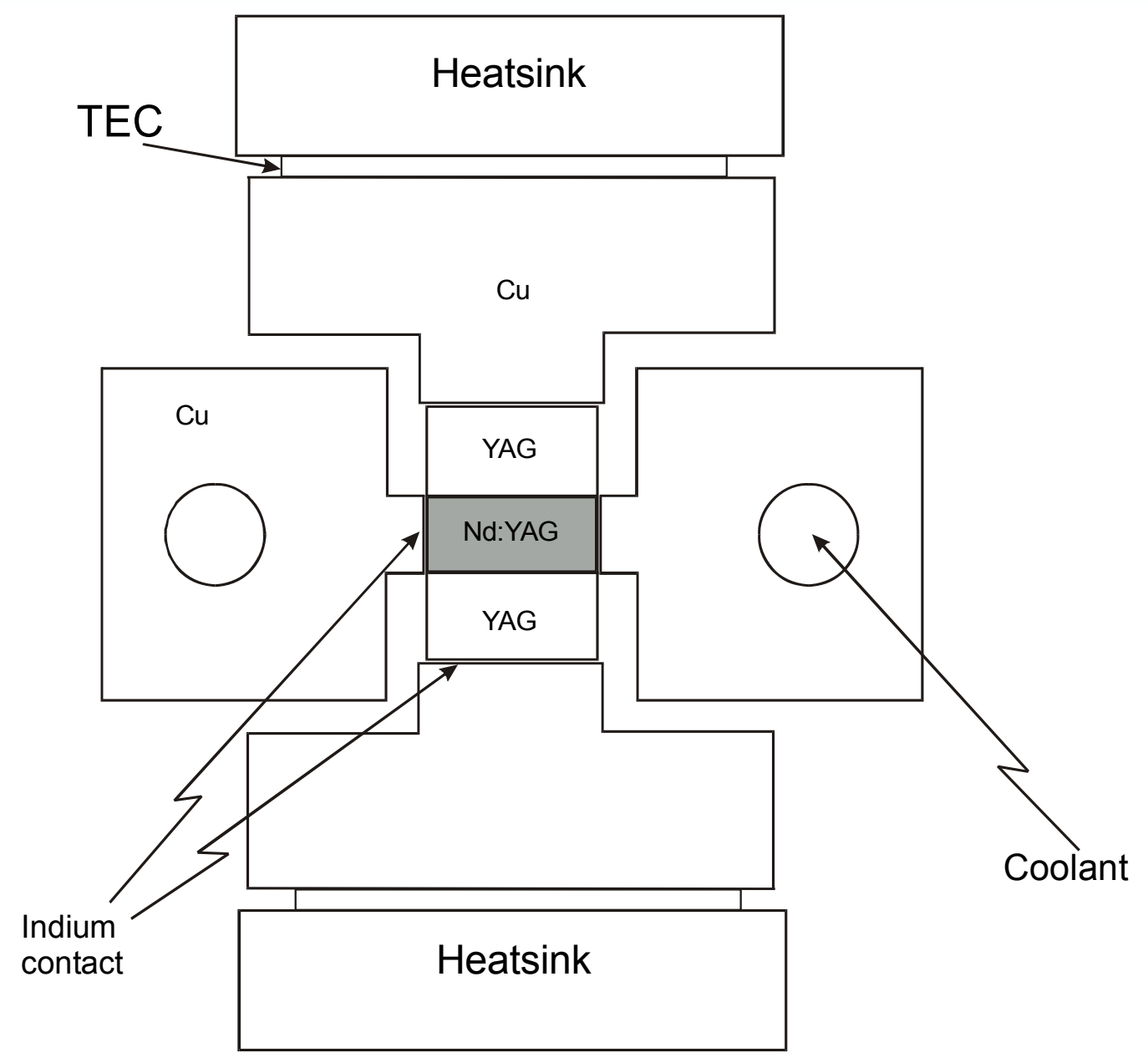




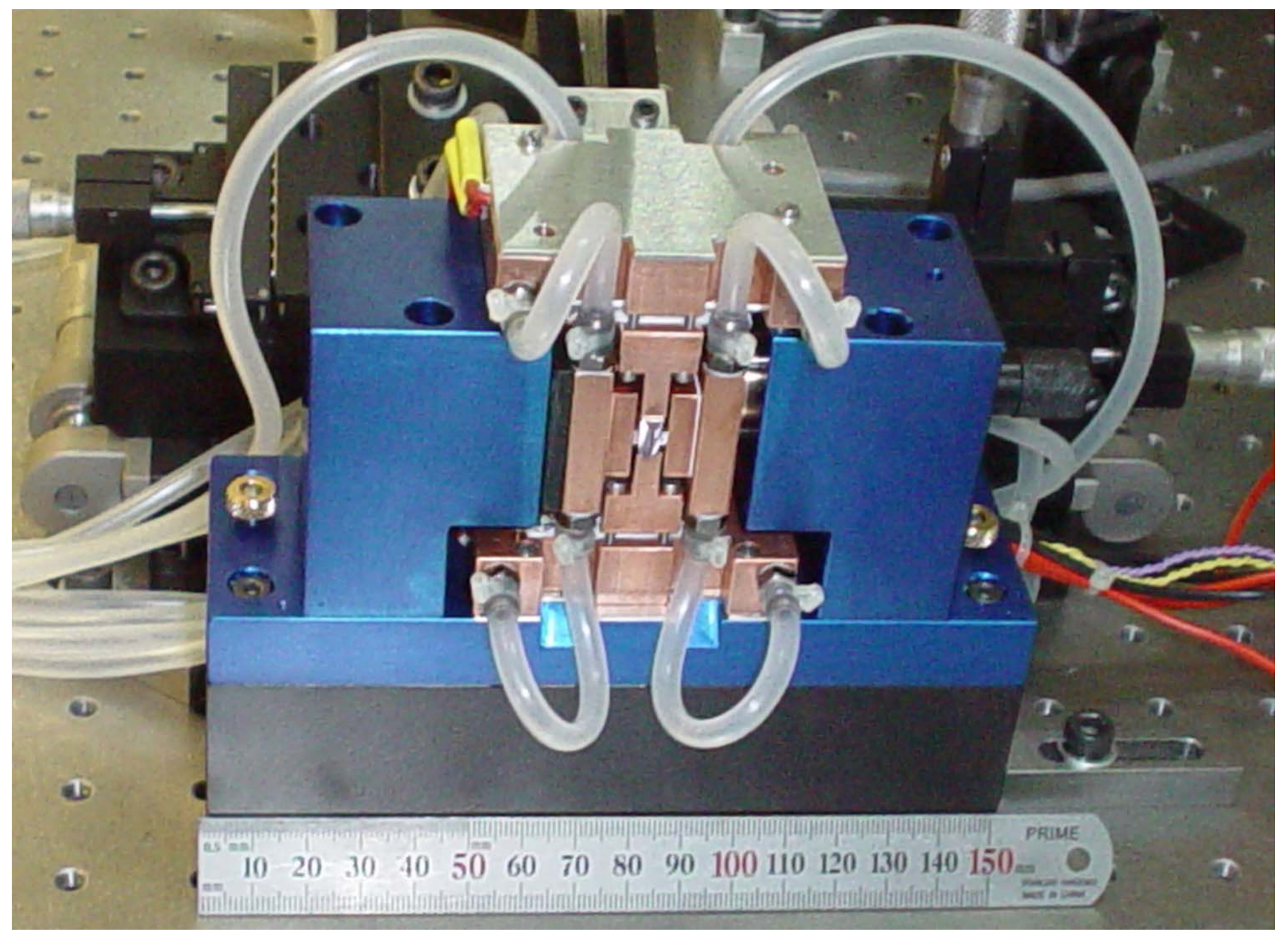

\section{* * THE UNIVERSITY \\ OF ADELAIDE \\ sents}




\section{Initial Laser Performance in Nd:YAG}

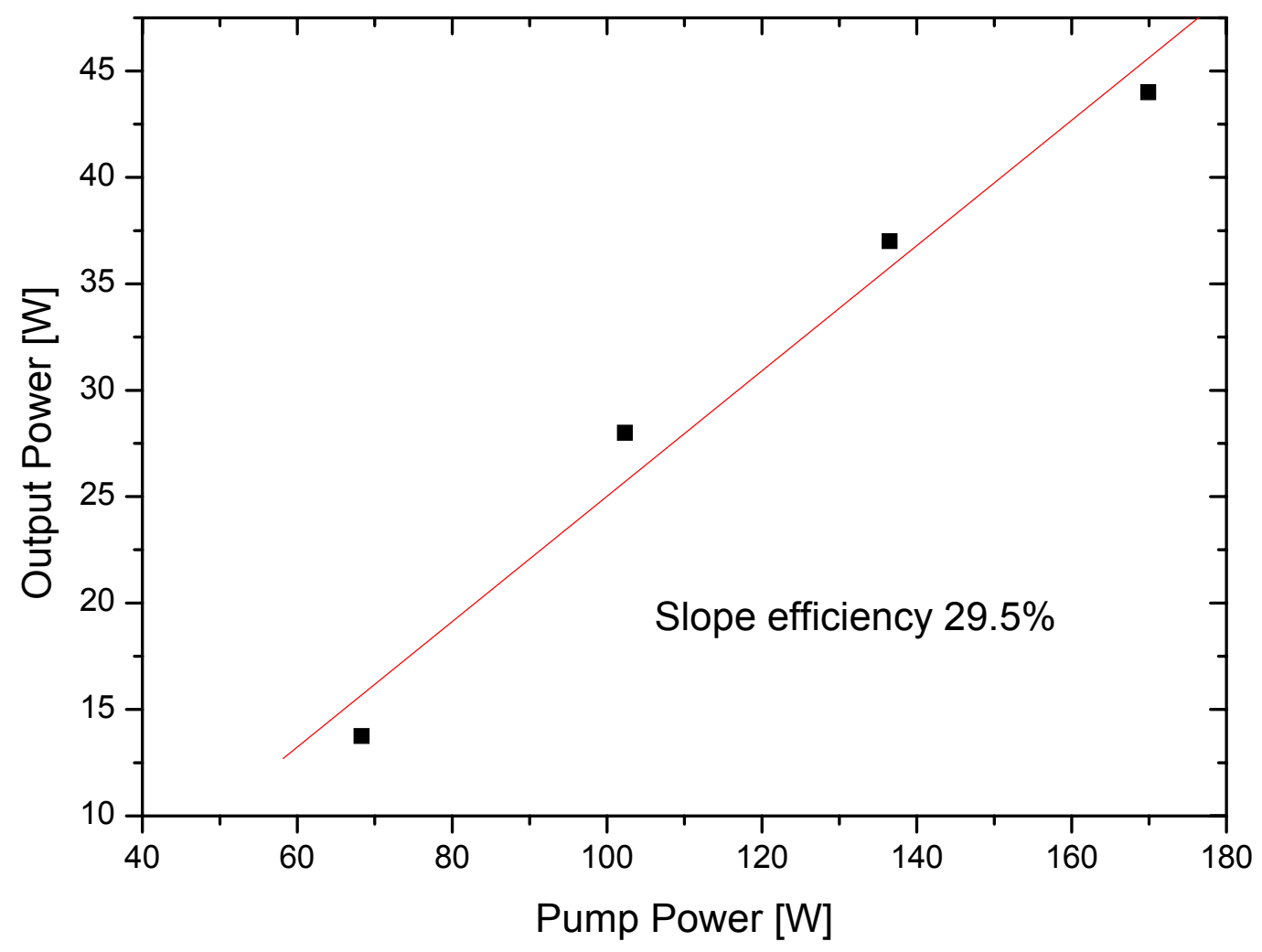

Approximately $90 \%$ pump light absorption in end-pumped slab 


\section{Composite slab design for Er:Yb:YAG}

- Ceramic

- Doped and undoped Er:Yb:YAG

- Doping concentrations easily changed

- Slab configuration based on success with Nd:YAG 


\section{Er:Yb:YAG laser radar system}

- New master oscillator under development

- NPRO (non-planar monolithic ring oscillator)

- Ceramic Er:Yb:YAG

- To be developed in collaboration with Innolight

- Injection seeded slave ring oscillator

- Ceramic composite slab slave as described 


\section{The DIAL program}

\section{(DIAL = differential absorption lidar)}

- Aim: Low-Cost profiling of water vapour up to top of boundary layer

- Provide water vapour concentrations for

- Quantitative precipitation forecasting, Bushfire danger assessment, fog prediction

- current technique - radiosondes, high recurrent cost, infrequent data

- $\quad 830 \mathrm{~nm}$ GaAs diode lasers (mature technology)

- Single mode limited to ca $0.5 \mathrm{~W}$ (Average power ca $0.5 \mathrm{~mW}$ - eyesafe!)

- Detector technology well developed (low-noise single photon)

- Wavelength control

- On-line laser (master oscillator) stabilised to peak of water resonance

- Off-line/ On-line difference frequency stabilised to $15 \mathrm{GHz}$

- Water resonance 6GHz width @ sea level 1GHz width @ 4km altitude

- Freq. stability of $\sim 20 \mathrm{MHz}$ adequate 


\section{Setup for DIAL}

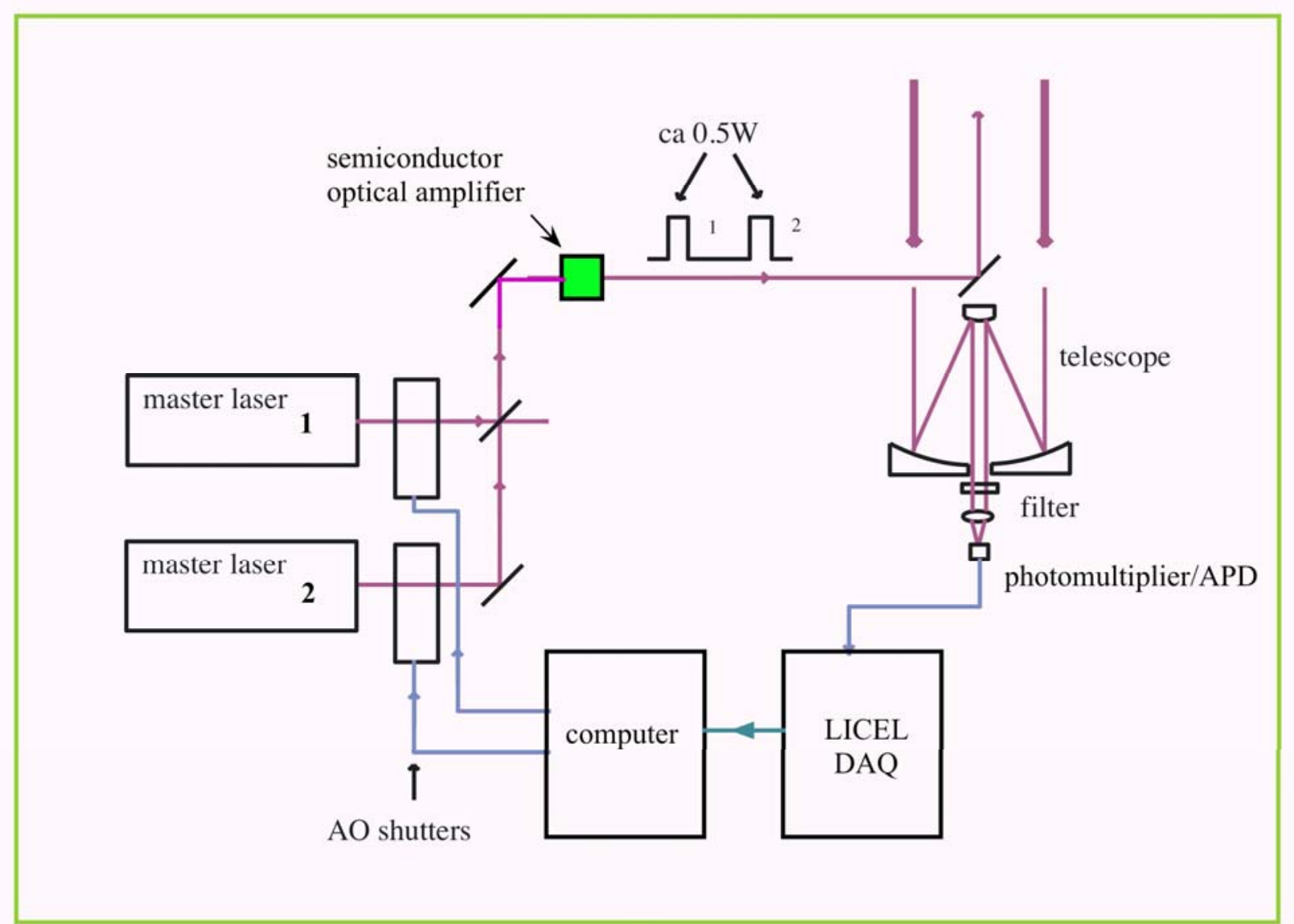




\section{Spectral properties of amplifier}

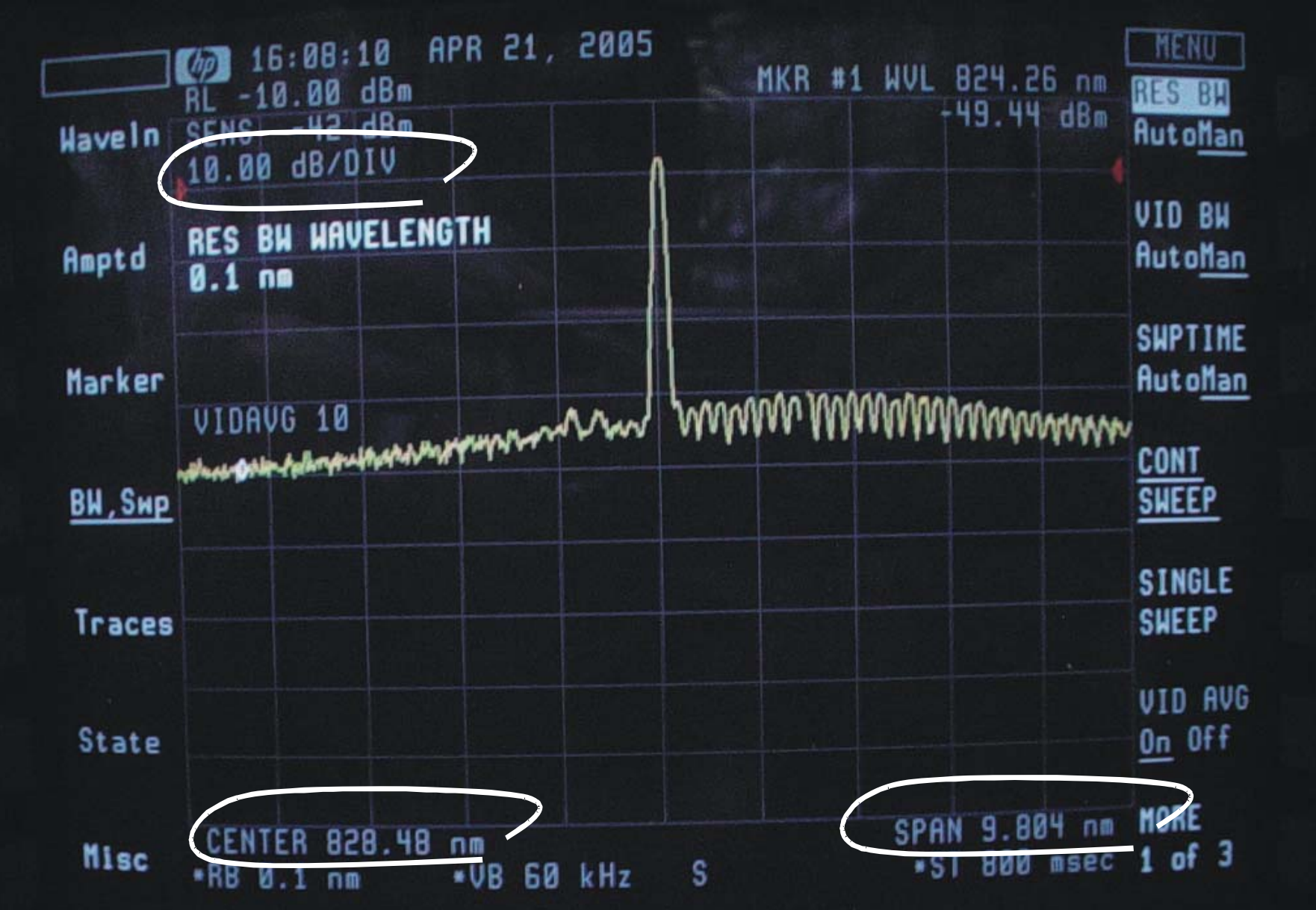




\section{Wavelength control of master lasers}

On-line laser stabilisation

- BLUE LOOP

Wavelength difference stabilisation

- GREEN LOOP

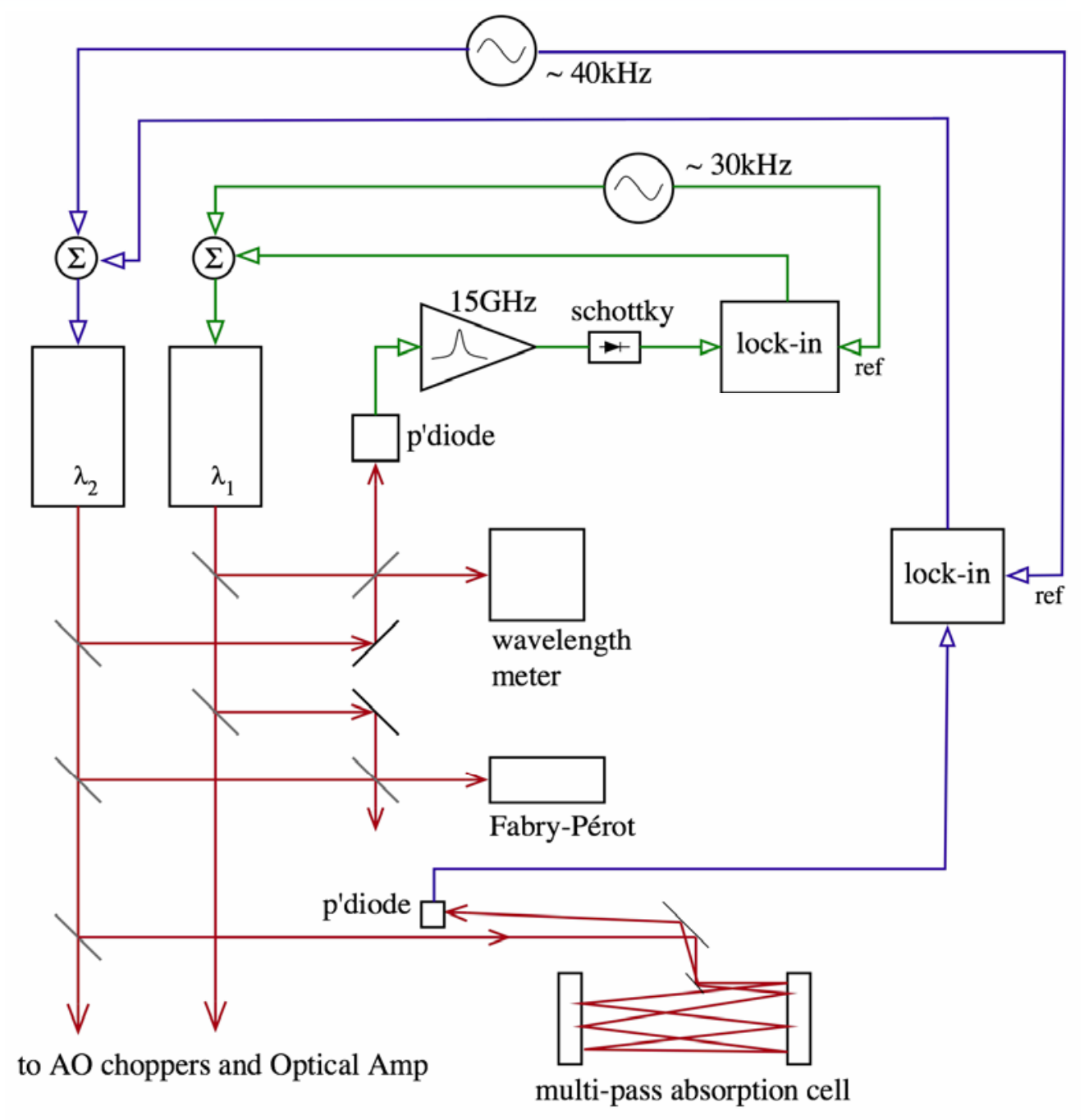




\section{Water resonances near 829nm}

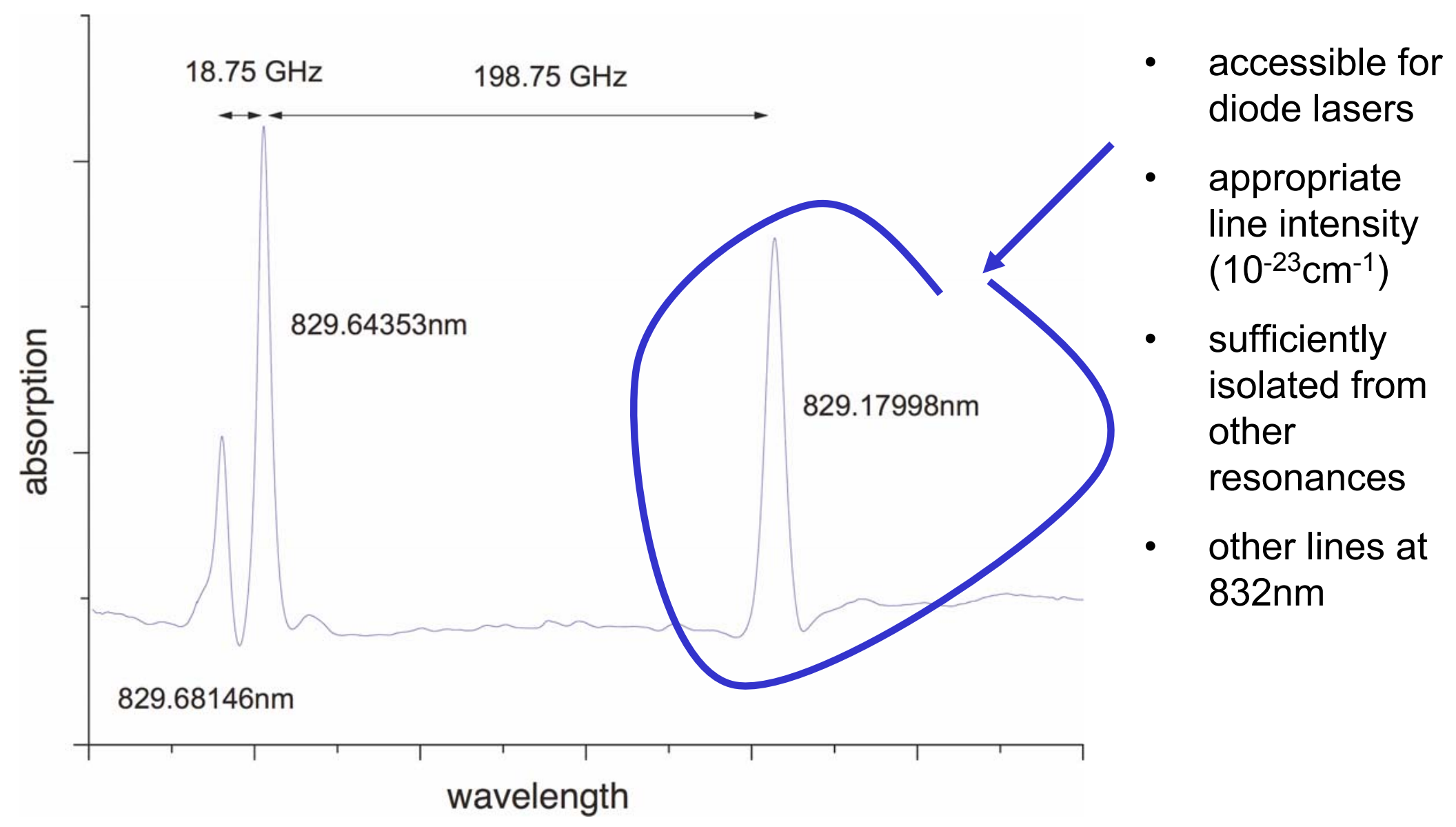




\section{Stabilization to water resonance $(832 \mathrm{~nm})$ \\ - error signal at lock-in output}
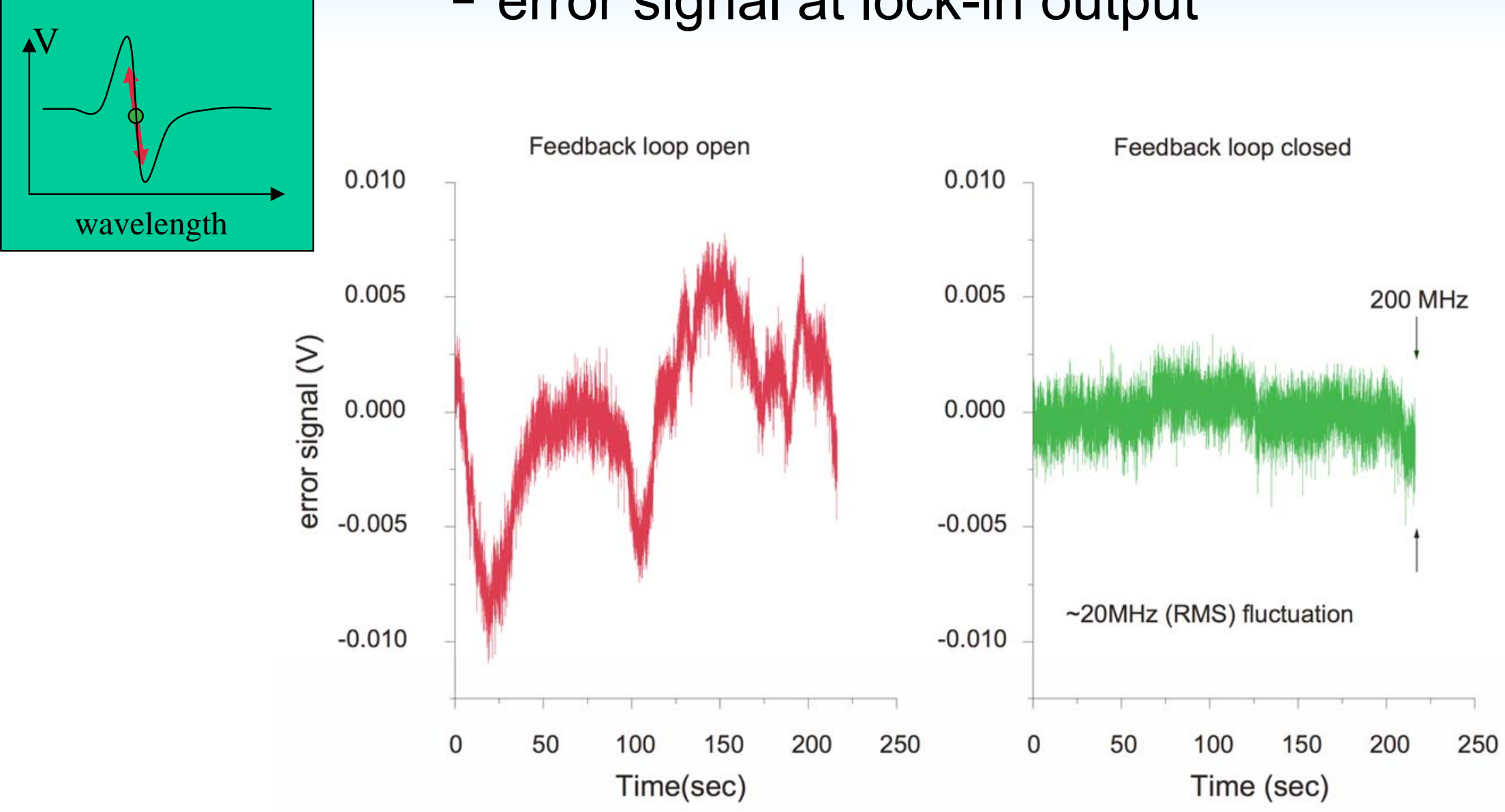


\section{Conclusion}

- Er:glass at $1.53 \mu \mathrm{m}$ is a useful approach for a simple, low average power eye-safe coherent laser radar, but is limited by thermal effects and damage in glass.

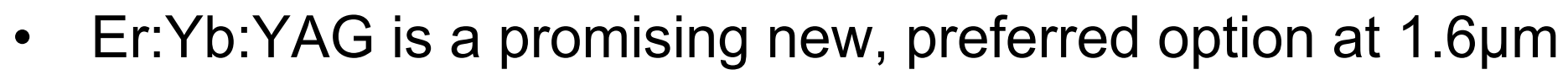
Design experience form Nd:YAG directly transferable

- Low cost alternatives to eye-safe incoherent sensing for short range $(<4 \mathrm{~km})$ applications using shorter wavelengths are feasible. 


\section{Producing a tophat pump distribution}

- How?

- Use a composite slab (doped \& undoped YAG layers)

- End-pumped for good efficiency

- Side-cooled zigzag slab

$\rightarrow$ Pump absorption is a tophat profile, thus minimizing thermally induced birefringence loss (even though diode-laser pump profiles typically produce Gaussian transverse profiles)

$\rightarrow$ Thermal lensing minimized by using a zigzag mode-path in the plane of cooling, and by controlling the heat flow in the orthogonal plane 


\section{Small-signal gain measurement proves bleaching of Erbium}

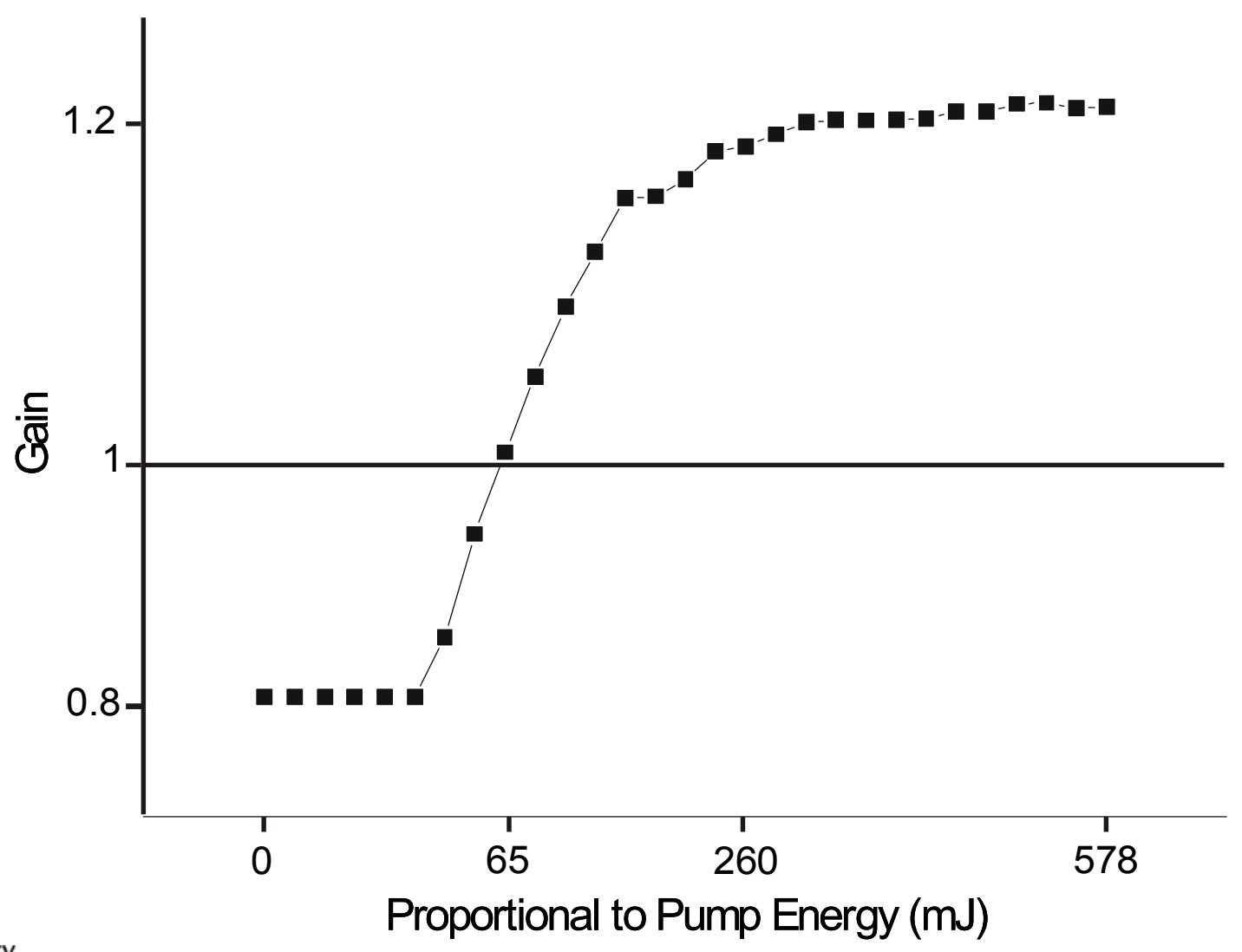

